\title{
Fragmentation, discrédit et politisation : un état des lieux du système éducatif péruvien
}

Damien Larrouqué

\section{OpenEdition}

1 Journals

Édition électronique

URL : https://journals.openedition.org/ries/8080

DOI : $10.4000 /$ ries. 8080

ISSN : 2261-4265

Éditeur

France Education international

\section{Édition imprimée}

Date de publication : 1 avril 2019

Pagination : $19-25$

ISBN : 978-2-85420-623-4

ISSN : $1254-4590$

Référence électronique

Damien Larrouqué, «Fragmentation, discrédit et politisation : un état des lieux du système éducatif péruvien », Revue internationale d'éducation de Sèvres [En ligne], 80 | avril 2019, mis en ligne le 01 avril 2021, consulté le 24 juin 2021. URL : http://journals.openedition.org/ries/8080 ; DOI : https://doi.org/ $10.4000 /$ ries.8080

Ce document a été généré automatiquement le 24 juin 2021.

(c) Tous droits réservés 


\title{
Fragmentation, discrédit et politisation : un état des lieux du système éducatif péruvien
}

\author{
Damien Larrouqué
}

1 D'après les données du système d'information des tendances éducatives en Amérique latine (SITEAL), le taux de scolarisation en primaire atteint au Pérou 97,3\%. Depuis 2005, la proportion des écoliers de 5 ans inscrits dans les établissements d'accueil de la petite enfance a bondi de $44 \%$, portant le taux de scolarisation en maternelle à $90 \%$ dix ans plus tard. De même, l'éducation secondaire reçoit désormais $10 \%$ d'élèves en plus qu'au début des années 2000 : le taux de scolarisation à ce niveau atteint $95 \%$ pour les adolescents de 12 à 14 ans et $76 \%$ pour ceux de deux ans leurs aînés ${ }^{1}$. S'ils attestent d'une quasi-universalisation de l'accès à l'éducation, ces bons chiffres n'en masquent pas moins le déficit de légitimité dont souffrent l'école publique et le système éducatif péruvien dans son ensemble. Comme ailleurs dans la région, une part importante de la classe moyenne, voire des classes populaires, tend à s'en détourner et à jeter son dévolu sur des offres éducatives alternatives.

2 Depuis la libéralisation de l'éducation par Alberto Fujimori (promulgation du décret $n^{\circ} 882$ en 1998), les établissements privés siphonnent année après année le contingent scolaire. Entre 2000 et 2014, la proportion d'élèves scolarisés dans le privé est passé de 14 à $26 \%$ (Balarin, 2016). Pourtant, la qualité de l'enseignement payant n'est en rien garantie. D'une manière générale, qu'elle soit dispensée par des institutions publiques ou privées, la formation éducative au Pérou se révèle de piètre facture. Cibles de toutes les critiques, les instituteurs pâtissent d'un manque de reconnaissance qui se traduit par un sentiment de relégation sociale et par des conditions financières et statutaires précaires. Au niveau institutionnel qui plus est, le ministère de l'éducation (Minedu) a toujours été considéré comme un «butin électoral ( (Oliart, 2011) permettant au parti vainqueur de récompenser ses plus fidèles affidés à coup de postes administratifs taillés sur mesure. La gestion s'avère donc relativement politisée. 
Depuis l'éviction du président conservateur Pedro Pablo Kuczynski en mars 2018, l'incrimination en janvier 2019 de son prédécesseur, le leader nationaliste de gauche, Ollanta Humala, puis le suicide en avril de l'ancien homme fort de la droite péruvienne, Alan García, sans compter l'arrestation aux États-Unis de l'ancien président Alejandro Toledo en juillet, tous les quatre poursuivis pour corruption, le Pérou est entré dans une période de grande incertitude politique. Ce climat de suspicion généralisée et de désaveu citoyen à l'égard de l'ensemble de la classe politique est susceptible de miner les promesses de développement ainsi que les efforts de rationalisation annoncés dans plusieurs domaines d'intervention publique, dont le système éducatif, lors du plan Perú 2021 (bicentenaire de l'indépendance).

Le constat que nous dressons dans cet article corrobore, en s'efforçant de les expliciter, les observations de chercheurs qui associent au système éducatif péruvien deux principales tares : « la corruption et la médiocrité » (Oliart, 2011). Objectivement, trois maux nous semblent affecter l'éducation dans ce pays andin de 32 millions d'habitants : une fragmentation exacerbée consécutive au boom incontrôlé de l'offre privée, une délégitimation profonde du métier d'instituteur liée à des conditions professionnelles peu enviables et, enfin, une gestion institutionnelle chaotique car soumise à un fonctionnement encore dominé par les logiques clientélistes.

\section{Une concurrence grandissante de l'école publique par une école privée dérégulée}

Depuis le milieu des années 1990, on constate une compétition croissante entre les établissements d'enseignement publics et privés au Pérou. L'attraction exercée par les seconds est de plus en plus forte; tant et si bien d'ailleurs que la plupart des professeurs enseignant dans l'éducation publique et l'écrasante majorité des fonctionnaires du ministère de l'éducation (Minedu) scolarisent leurs propres enfants dans des établissements sous (ou sans) contrat. À l'instar du processus de fragmentation éducative à l'œuvre dans d'autres régions du monde, la survalorisation de l'éducation privée trouve en partie son origine dans une volonté de différenciation sociale. C'est moins la qualité que l'exclusivité qui est recherchée. L'école péruvienne se présente de plus en plus comme une école de classe : l'éducation nationale est « une institution pauvre pour les pauvres » (Oliart, 2011).

6 Le boom de l'enseignement privé est particulièrement à l'œuvre dans les villes et singulièrement dans la capitale. En 2008, concernant le seul niveau primaire, la province de Lima comptait plus de 3350 écoles de gestion privée contre un peu moins de 940 établissements d'enseignement public, selon les informations fournies par Juan Ansion, sociologue rattaché à l'Université pontificale catholique du Pérou². Si le processus de « migration éducative » se poursuit au même rythme que dans les années 2000, son collègue de l'Institut d'études péruviennes (IEP), Ricardo Cuenca, estime que trois élèves sur quatre seront scolarisés dans le privé en 2020. Cette concurrence provoque une énorme fragmentation éducative entre régions, entre types de gestion et entre catégories sociales : l'éducation péruvienne fonctionne désormais à plus de trois vitesses (Muelle, 2018).

7 Du reste, le manque criant de régulation constitue le principal problème associé à l'enseignement privé dans la région. En l'occurrence, le ministère de l'éducation 
péruvien s'est montré, des années durant, incapable d'enrayer la prolifération de ces établissements et surtout de vérifier la qualité des cours dispensés à leurs élèves. C'est ce que María Balarin a appelé la «privatisation par défaut » (Balarin, 2016). Malgré une amélioration au cours des dernières années, l'accès à l'information publique reste parcellaire. Sans être emmuré dans cette traditionnelle culture du secret (secretismo) qui l'a longtemps caractérisé, le Minedu éprouve encore des difficultés à communiquer des données fiables. Et pour cause, si l'on estime qu'environ 8700000 élèves relèvent de l'éducation obligatoire dans le pays ${ }^{3}$, le ministère a en réalité très peu de visibilité sur le nombre exact d'élèves inscrits dans des établissements sous (ou sans) contrats. Concrètement, ni le Minedu, ni les chercheurs et encore moins les parents d'élèves concernés n'ont accès à une base de données qui répertorierait des informations aussi précieuses que le coût de la scolarité, la dimension lucrative ou non des établissements, leur accréditation potentielle, les qualifications des enseignants ou leurs conditions de travail - lesquelles s'avèrent souvent encore plus précaires que dans le public. En somme, les établissements privés évoluent dans un no man's land juridique et financier (Balarin, 2016). Cette absence de régulation a été accentuée par le processus de régionalisation éducative initié en 2004, ainsi que l'ont mis en lumière A. M. Manrique Linares et C. del Mastro.

8 De manière générale, la qualité de l'enseignement reste très mauvaise. Intégré dès 2000 aux enquêtes du Programme international pour le suivi des acquis des élèves (PISA) produites par l'Organisation de coopération et de développement économiques (OCDE), le Pérou a toujours figuré à la dernière place, avant de remonter depuis 2015 dans le peloton de queue du classement international. Régulièrement relayées par les médias nationaux, ces évaluations négatives retombent souvent sur les enseignants qui endurent dans l'opinion un discrédit persistant (et en partie justifié).

\section{Instituteur, une profession en mal de reconnaissance sociale et financière}

9 Au Pérou, les instituteurs sont accusés de porter la responsabilité du marasme éducatif contemporain. Leur propre niveau scolaire est réputé passable pour ne pas écrire médiocre. Ces lacunes ont éclaté au grand jour lors de la mise en œuvre de la très décriée loi de carrière publique magistérielle (CPM), sur laquelle se sont penchés L. M. Saravia et M. López de Castilla. Approuvée en juillet 2007 durant le second mandat d'Alan García (2006-2011), la CPM est associée à la figure charismatique de l'inamovible ministre de l'éducation José Antonio Chang Escobedo - lequel aura connu pendant cinq ans une longévité exceptionnelle à la tête du Minedu, quand ses prédécesseurs étaient remplacés en moyenne tous les ans. Inspirée du modèle méritocratique chilien, la CPM a accouché aux forceps de la pratique de l'évaluation continue. Réalisés tous les trois ans et sanctionnés par l'exclusion du professorat suite à deux échecs successifs ${ }^{4}$, ces examens ont été accueillis de manière au mieux «désagréable» et au pire «traumatique » par une très grande majorité de professeurs, qui ont vu leurs piètres résultats faire les gros titres de la presse péruvienne. Combattue bec et ongles par le SUTEP, le puissant syndicat unitaire d'extrême gauche qui s'enorgueillit de rassembler quelque 300000 adhérents, soit $85 \%$ du corps enseignant ${ }^{5}$, la CPM a été abrogée par le gouvernement socialiste d'ollanta Humala (2011-2016). Au grand dam des instituteurs les plus réticents à voir leur travail régulièrement évalué, la Loi de réforme 
magistérielle (LRM) qui lui a succédé, a pourtant renforcé la prétention méritocratique du système d'enseignement $t^{6}$. Notons que les vicissitudes de cette réforme révèlent, à la fois, une grande résistance au changement de la part des acteurs engagés dans l'éducation péruvienne et une relative instabilité des politiques publiques dans ce pays (Lynch, 2006).

10 Depuis une quinzaine d'années, notons que les instituteurs ont pu apprécier une revalorisation financière substantielle. Sous le gouvernement d'Alejandro Toledo (2001-2006) notamment, ils ont enregistré un quasi-doublement de leur salaire. En moins de cinq ans, leur revenu a augmenté de 500 sols, soit d'environ 130 euros, pour s'établir actuellement autour de 1100 sols, ce qui ne représente toutefois guère plus de 300 euros mensuels. Leur insatisfaction professionnelle demeure aussi la plus aiguë de la région, comme l'ont démontré Javier Murillo de l'Université Autonomede Madrid et son collègue de l'Université Alberto Hurtado du Chili. Leur statut est précaire et ils sont souvent obligés de multiplier les charges d'enseignements entre plusieurs établissements. Leur intégrité s'en trouve parfois compromise. Ainsi, pour arrondir les fins de mois et sans que la pratique soit pour autant monnaie courante, il arrive que d'aucuns se livrent à des actes de prévarication: il nous a ainsi été rapporté que certains instituteurs monnayent auprès des parents la correction des devoirs de leurs enfants ou proposent des cours privés à partir de matériels pédagogiques entièrement plagiés sur internet ${ }^{7}$.

$11 \mathrm{Au}$ demeurant, pour ce qui est du versement de bakchichs (coimas), le système éducatif est de loin, derrière la police, l'institution la moins corrompue de tout l'appareil d'État péruvien selon les travaux d'un couple de chercheurs de l'Université du Pacifique publiés en 2011. Si ces perceptions négatives doivent donc être relativisées, l'inféodation de l'administration publique au pouvoir politique continue de nuire à la cohérence et la continuité de l'action publique.

\section{Une gestion institutionnelle symptomatique de la versatilité de l'action politico-administrative}

Dans sa thèse de doctorat soutenue à l'université de Bath en 2005 et intitulée «Radical discontinuity. A study of the role of education in the Peruvian state and of the institutions and cultures of policy making in education ", María Balarin a mis en lumière plusieurs facteurs de "discontinuité " au sein du Minedu: ils concernent notamment le personnel administratif, le cadre juridique et les interférences institutionnelles avec d'autres ministères.

13 Le premier enjeu est relatif aux ressources humaines. Selon cette sociologue, il est commun que les instituteurs achèvent leur carrière en entrant dans les rangs du Minedu. Ils occupent alors des postes de complaisance mal payés mais perçoivent, cependant, des revenus substantiellement plus élevés que ceux qu'ils recevaient en tant qu'enseignants. Par là même, ils obtiennent également la sécurité de l'emploi jusqu'à leur retraite. Depuis les années 1970, cette logique de " pantouflage administratif » en forme de couronnement d'une carrière ingrate effectuée dans l'enseignement a engendré l'organisation macrocéphale et portée sur le dilettantisme qui est tant décriée aujourd'hui (Oliart, 2011). Obscurs, les critères d'ascension professionnelle sont, de surcroît, laissés à l'appréciation discrétionnaire des cadres de l'institution. Arrivés à 
leurs postes en même temps que le ministre qui les a désignés et dont ils deviennent les obligés, ces derniers touchent des salaires relativement élevés - longtemps financés à partir des fonds alloués par les créanciers internationaux -, mais dépendent de contrats précaires renouvelables chaque année.

Au-delà du fait que cette différence de statut entre fonctionnaires mal payés et cadres contractuels très bien rémunérés ne va pas sans créer des frictions internes, ces logiques clientélistes ont abouti à une «bureaucratie de pactes " (bureaucracy of pacts) profondément stérile. De fait, compte tenu de l'anémie politico-partisane d'une part (absence d'un programme politique cohérent à l'échelle nationale) et de l'instabilité gouvernementale d'autre part - les ministres sont révocables à tout moment-, le nouveau ministre de l'éducation tâche de faire ses preuves en conduisant, le plus rapidement possible et en toute indépendance, le projet qu'il a lui-même défini comme prioritaire. À cette fin, il s'entoure de ses partisans avec lesquels il noue, en quelque sorte, un pacte politico-administratif. Or, dès lors que le ministre est remplacé (en moyenne chaque année durant les présidences de Fujimori et de Toledo), le pacte est rompu et un nouveau projet prioritaire est lancé par l'intermédiaire d'une nouvelle équipe. Malgré l'arrivée au pouvoir du gouvernement de gauche incarné par ollanta Humala (2011-2016), la traditionnelle cooptation politique a perduré. Le turn- over administratif demeure très important au sein du Minedu, ce qui n'est pas sans affecter la continuité de l'action publique. Au cours des huit dernières années, le Minedu a connu six ministres différents.

Par ailleurs, la culture institutionnelle est si singulière qu'il n'y a pas de transmission de savoir-faire entre deux administrations. En conséquence, les programmes éducatifs se répètent ou entrent en contradiction inlassablement. En substance et concernant les ressources humaines, la politiste déplore le défaut de sélection méritocratique des agents publics, l'irrationalité des processus d'ascension professionnelle ainsi que l'absence d'une classe de fonctionnaires de type intermédiaire qui, garante de la mémoire institutionnelle, aurait à charge de faire l'articulation entre la décision politique et l'exécution administrative.

16 Pour ce qui est du cadre légal, María Balarin regrette encore que les procédures d'approbation soient sources d'embrouillaminis bureaucratiques, car très rigides et soumises à l'aval de plusieurs personnes qui constituent autant d'acteurs-veto (veto players). Cette difficile coordination s'avère d'autant plus exacerbée que le processus de régionalisation a débouché sur une armature juridico-institutionnelle inextricable : les doublons se multiplient et les responsabilités entre différents niveaux de gouvernement se télescopent. L'inertie administrative trouve enfin son origine dans les problèmes de collaboration avec d'autres ministères. Très concrètement, le ministère de l'économie et des finances (MEF) est souvent accusé d'interférer, par défiance, en défaveur des initiatives les plus onéreuses proposées par le Minedu. Dans un tel contexte, les politiques éducatives sont portées à ne rester que purement rhétoriques (Cuenca, 2012).

\section{Les défis contemporains de l'éducation péruvienne}

17 Au terme de ce rapide état des lieux, il nous semble que quatre défis s'imposent au système éducatif péruvien. Or, seul le troisième a été considéré dans le plan sectoriel 
pluriannuel d'éducation qui a fixé, en 2016, les grandes orientations stratégiques pour les cinq années à venir.

En premier lieu, la revalorisation de l'éducation publique ne peut se passer d'un investissement plus conséquent en sa faveur. Selon les chiffres de la Banque mondiale, le Pérou y consacre 3,9\% de son produit intérieur brut (PIB), contre 4,4 \% en Colombie, $5 \%$ au Chili et $5,2 \%$ au Mexique, pour ne citer que ses trois partenaires commerciaux au sein de l'Alliance du Pacifique.

19 En second lieu, la régulation des structures d'enseignement privé constitue une condition sine qua non de l'amélioration de l'éducation péruvienne. La prolifération de ces établissements, sans cadre juridique clair ni contrôle de la part des autorités, contribue à miner la crédibilité de l'ensemble du système et porte préjudice aux familles les moins informées, lesquelles se retrouvent à s'endetter pour des formations dont la médiocrité n'a d'égale que leur coût abusif.

En troisième lieu, si le changement de perception à l'égard du métier d'instituteur passe très certainement par un renforcement de la formation - tel que le prévoit le plan 2016-2021 -, il semble opportun que cette réévaluation des exigences académiques s'accompagne également d'une revalorisation salariale et d'une amélioration des conditions de travail. Mieux payer les instituteurs permettrait, en toute logique, de juguler les comportements répréhensibles, ou tout du moins peu éthiques, auxquels se livrent certains.

Enfin, sur le plan institutionnel, le défi le plus important reste vraisemblablement celui de la rationalisation du fonctionnement administratif. L'introduction de dispositifs de sélection méritocratique des agents publics doit permettre de dépolitiser le ministère, où la mémoire institutionnelle, reposant sur l'agrégation des savoir-faire individuels et collectifs acquis, se transmet moins qu'elle ne réinitialise de manière perpétuelle. Sous cette optique, la continuité de l'action publique s'avère illusoire.

\section{BIBLIOGRAPHIE}

BALARIN M. (2016) : « La privatización por defecto y el surgimiento de las escuelas privadas de bajo costo en el Perú. ¿Cuáles son sus consecuencias? ", Revista de la Asociación de Sociología de la Educación-RASE, vol. 9, n² 2, p. 181-196.

CUENCA R. (2012) : « Desencuentros entre el discurso del derecho a la educación y las políticas educativas en el Perú de la década del 2000 », Documento de Trabajo IEP, n 170, p. 1-76.

LYNCH N. (2006) : Los últimos de la clase. Aliados, adversarios y enemigos de la reforma educativa en el Perú, Lima : Fondo Editorial de la UNMSM.

MUELLE L. (2018) : « Desigualdades regionales y sociales del rendimiento escolar al término la educación primaria en el Perú ", Revista peruana de investigación educativa, n 10, p. 127-157.

OLIART P. (2011) : « Mediocridad y corrupción : los enemigos de la educación pública », in L. Pásara (dir.), Perú ante los desafíos del siglo XXI, Lima : Fondo Editorial PUCP, p. 295-325. 


\section{NOTES}

1. Pour en savoir plus, voir le site du SITEAL, rubrique Perfil País: Perú. Actualisé en mai 2019.

2. Pour des raisons de format, ne sont ici citées que les références bibliographiques deux fois convoquées a minima ou les plus récentes. Les autres travaux mobilisés peuvent néanmoins être communiqués sur demande (damien.larrouque@sciencespo.fr). Nous renvoyons le lecteur curieux à l'excellente revue annuelle intitulée Revista peruana de investigación educativa [http:// revistas.siep.org.pe/RPIE] (NdA).

3. INEI, Número de alumnos matriculados en el sistema educativo nacional, según departamento, 2008-2017, Lima, 2018. Consultation en ligne.

4. Articles $n^{\circ} 24,28$ et 29 de la loi $n^{\circ} 29602$ du 11 juillet 2007.

5. D'après $\mathrm{N}$. Lynch, sociologue et ancien ministre de l'éducation sous gouvernement Toledo (2011-2012), ces chiffres sont vraisemblablement surévalués. À la date de sa légalisation en 1985 , le syndicat unique péruvien représentait $74 \%$ du magistère (Lynch, 2006).

6. Chap. VI de la loi ${ }^{\circ} 29944$ du 23 novembre 2012.

7. Propos recueillis le 8 avril 2013 à Lima, auprès d'un professeur de mathématiques en détachement au SUTEP.

\section{INDEX}

Mots-clés : politique éducative, enseignement privé, privatisation de l'éducation, qualité de l'enseignement, enseignant

Palabras claves : política educacional, enseñanza privada, privatización de la educación, calidad de la enseñanza, docente

Keywords : educational policy, private education, privatization of education, teaching quality, teachers

Index géographique : Pérou

\section{AUTEUR}

\section{DAMIEN LARROUQUÉ}

Damien Larrouqué est chercheur post-doctoral auprès de l'Institut des affaires publiques (INAP) de l'Université du Chili et docteur associé à l'Observatoire politique de l'Amérique latine et des Caraïbes (OPALC) de Sciences Po. Lauréat de la bourse Fondecyt (2019-2021) attribuée par la Commission nationale de recherche scientifique et technologique (Conicyt) dans la catégorie "sciences politique et juridique ", il travaille sur les transformations de la haute fonction publique dans le Cône sud (Chili, Argentine, Uruguay). Outre les questions éducatives en Amérique latine, il s'intéresse également aux évolutions des partis politiques dans la région. Courriel : damien.larrouque@sciencespo.fr 